\title{
Graded Poisson Lie Structures on Classical Complex Lie Groups
}

\author{
G.E. Arutyunov \\ Steklov Mathematical Institute, Vavilov 42, GSP-1, 117966, Moscow, Russia \\ E-mail: arut@qft.mian.su
}

Received: 21 November 1994/Accepted: 11 September 1995

\begin{abstract}
The external algebra over holomorphic first order differential forms on a complex Lie group $G$ is endowed with the structure of a graded Poisson Lie algebra. This structure is introduced via graded bicovariant brackets that are shown to be in one to one correspondence with $G$-invariant tensors of special symmetry. Complete classification of graded Poisson Lie structures defined by homogeneous brackets is obtained for the case of classical complex Lie groups.
\end{abstract}

\section{Introduction}

The aim of this paper is to classify homogeneous Poisson Lie structures on the algebra of differential forms on a classical complex Lie group. This investigation is inspired by the issue of differential calculus on quantum groups for which the structures in question appear in the semiclassical approximation. Such a connection between graded Poisson Lie structures and differential calculus on quantum groups reflects the general Faddeev concept [1] that all objects in the quantum group theory should appear as the result of deformation (quantization) of appropriate Poisson structures [2-6].

Note that the notion of graded Poisson Lie structures [7] and the studies of their special examples $[8-10]$ were stimulated by the papers $[11,12]$ where it was proved that bicovariant differential calculus on quantum groups can be supplied with the structure of a graded (super) Hopf algebra. At the semiclassical level this fact indicates the existence of graded Poisson Lie structures and the close analogy between such structures and Poisson Lie groups. Namely, a graded Poisson Lie structure governs the deformations of the external algebra in the same way as Poisson Lie group structures determine the deformation of the function algebra in the category of Hopf algebras. These deformed algebras can be viewed as algebras of external forms (functions) on a corresponding quantum group. 
The recent developments ${ }^{1}$ of bicovariant differential calculi for various quantum groups follow the general scheme elaborated by Woronowicz [13, 14]. Recall that Woronowicz starts with the function algebra $\mathscr{A}$ on a quantum group and introduces a bicovariant bimodule $\Omega$, i.e., a bimodule over $\mathscr{A}$ supplied with two coactions $\Delta_{r}: \Omega \rightarrow \Omega \otimes \mathscr{A}$ and $\Delta_{l}: \Omega \rightarrow \mathscr{A} \otimes \Omega$ satisfying the set of axioms [14]. Then a first order differential calculus is defined as a pair $(\Omega, d)$, where $d: \mathscr{A} \rightarrow \Omega$ is a nilpotent mapping obeying the Leibnitz rule. It is proved that any first order differential calculus can be lifted to higher order differential forms.

Aside from the obvious theoretical meaning the deformation approach also has practical significance. The point is that there is a number of open questions in Woronowicz's theory, which arise when one incorporates information specifying a quantum group into the above scheme. For instance, the explicit description of defining relations of quantum external algebras appears to be a nontrivial task [15-18]. Another complicated problem is to check for these algebras the Diamond Condition [17-22]. Concerning simple quantum groups one finds that the set of generators for $\Omega$ involves additional elements that have no natural classical counterparts [23]. All these phenomena are usually treated as being of specific quantum nature. At the same time our analysis [7-9] reveals the similar phenomena just at the level of Poisson Lie structures. Hence, one has a chance to clarify these questions by using the powerful tools of classical (non-quantum) group theory.

Let us briefly describe the main points of our construction as well as the structure of the paper. Section 2 collects the definitions concerning Poisson Lie groups and classical matrix Lie algebras. In Sect. 3 we define on the external algebra $\Omega$ over the cotangent bundle of a Lie group $G$ a special bilinear operation called the bracket. The right (left) translations of $G$ are lifted to $\Omega$. Following Woronowicz we require bicovariance, i.e., covariance under right and left translations to be the main characteristic property of this bracket. Further we use the bicovariant bracket to define the structure of a graded Poisson Lie algebra.

In Sect. 4 the notion of bicovariant bracket is investigated. We indicate that all bicovariant brackets on $\Omega$ are in one to one correspondence with $G$-invariant tensors with special symmetry. Having in mind the connection with bicovariant differential calculi on corresponding quantum groups we restrict ourselves to the case of a homogeneous bicovariant bracket. In Sect. 5 we derive necessary conditions on this bracket to supply $\Omega$ with the structure of a graded Poisson Lie algebra. Here we note that the main obstruction arises from the Jacobi identity involving only odd generators of $\Omega$.

In Sect. 6, by using the concept of a fundamental Poisson bracket we prove our main result (Theorem 3 ) about the classification of graded Poisson Lie structures determined by homogeneous brackets. We find that if $G$ is of type $A_{n-1}, n>2$, then there exist four such structures and if $G$ is one of types $B_{n}, C_{n}$ or $D_{n}$ (we exclude the classical isomorphisms), then the graded Poisson Lie structure determined by a homogeneous bracket does not exist. The case of $A_{1}$ is distinguished since it admits two structures in question. The consequences of this theorem are discussed in the Conclusion.

1 The list of references can be found in [8]. 


\section{Preliminaries and Notation}

This section serves to fix the notation. ${ }^{2}$

Let $G$ be a Lie group with a Lie algebra $\mathscr{G}$ and let $\mathscr{A}$ be the algebra of holomorphic functions on $G$. This is a Hopf algebra with the comultiplication $\Delta$, the counit $\varepsilon$ and the antipod $S$.

A Lie group is called a Poisson Lie group, if $\mathscr{A}$ is supplied with a Poisson bracket $\{,\}_{G}$ satisfying the relation

$$
\Delta\{f, h\}_{G}=\{\Delta f, \Delta h\}_{G \times G},
$$

where $G \times G$ is equipped with the product Poisson structure.

Let $T=\left(t_{i}^{j}\right)_{i, j=1}^{N}$ be an exact holomorphic representation of $G$ in a vector space $V$ and $\tau$ be the corresponding representation of $\mathscr{G}$. It is suitable to define a Poisson structure on $G$ in terms of matrix coefficients $t_{i}^{j}$. If $G$ is a semisimple connected Lie group, then for this set of generators a Poisson bracket on $\mathscr{A}$ defining the structure of a Poisson Lie group has the form [2]

$$
\left\{T_{1}, T_{2}\right\}=\left[(\tau \otimes \tau) r, T_{1} T_{2}\right],
$$

where we have used the standard tensor notation: $T_{1}=T \otimes I, T_{2}=I \otimes T$, etc., to suppress matrix indices. Here $r \in \wedge^{2} \mathscr{G}$ is the classical $r$-matrix, i.e. a solution of the modified Yang Baxter Equation (mYBE) that requires an element

$$
[[r, r]] \stackrel{\text { def }}{=}\left[r_{12}, r_{13}+r_{23}\right]+\left[r_{13}, r_{23}\right] \in \wedge^{3} \mathscr{G}
$$

to be invariant under the adjoint action of $\mathscr{G}$.

For simple Lie algebras it holds

Proposition 1 [2]. If $\mathscr{G}$ is a simple Lie algebra, then there exists a unique (up to a multiplicative constant) invariant element in $\wedge^{3} \mathscr{G}$. In a basis $\left\{e_{\mu}\right\}$ of $\mathscr{G}$ it can be presented in the form $\left[K_{13}, K_{23}\right]$, where $K=g^{\mu v} e_{\mu} \otimes e_{v}$ and $g^{\mu \nu}$ is the inverse of the Killing tensor.

Hence, in the case of a simple $\mathscr{G}$ the mYBE reads $[[r, r]]=-\alpha\left[K_{13}, K_{23}\right]$, where $\alpha \neq 0$ is a complex parameter.

Now we list some conventional notations concerning classical series of Lie algebras. Let $\mathscr{G}$ be a simple Lie algebra corresponding to one of the classical series $A_{n-1}$ $(s l(N), N=n), B_{n}(s o(N), N=2 n+1), C_{n}(s p(N), N=2 n)$ or $D_{n}(s o(N), N=2 n)$. In fundamental representation

$$
\begin{gathered}
\mathscr{G}=\{X \in \operatorname{Mat}(N, \mathbf{C}) \mid \operatorname{tr} X=0\} \quad \text { for } A_{n-1} ; \\
\mathscr{G}=\left\{X \in \operatorname{Mat}(N, \mathbf{C}) \mid X^{t}=-C X C^{-1}\right\} \quad \text { for } B_{n}, C_{n}, D_{n} .
\end{gathered}
$$

Here $X^{t}$ means the transpose and $C=\left(C_{i j}\right)$ is an $N \times N$ metric: $C_{i j}=\varepsilon_{i} \delta_{i^{\prime} j}$, $i^{\prime}=N+1-i$ such that all $\varepsilon_{i}=1$ for $B_{n}$ and $D_{n}$, and $\varepsilon_{i}=1, i=1, \ldots, N / 2$, $\varepsilon_{i}=-1, i=N / 2+1, \ldots, N$ for $C_{n}$. By definition, we put $C^{-1}=\left(C^{i j}\right)$.

Throughout the paper Latin and Greek letters are used to run the sets $\{1, \ldots, N\}$ and $\{1, \ldots, \operatorname{dim} \mathscr{G}\}$ respectively.

\footnotetext{
2 The nice introduction to Poisson Lie groups can be found in [24].
} 


\section{Definition of Graded Poisson Lie Structure}

Let $G$ be a complex matrix Lie group and $\Omega^{1}=T^{*} G$ be the cotangent bundle over $G$. Introduce a $\mathbf{Z}$-graded vector space

$$
\Omega=\bigoplus_{n \geqq 0} \Omega^{n}
$$

where $\Omega^{0}=\mathscr{A}$ and $\Omega^{n}$ is the space of differential $n$-forms. $\Omega$ is a bimodule over $\mathscr{A}$. In the following we treat elements of $\mathscr{A} \subset \Omega$ as forms of order zero. For the sake of shortness we will denote the product in $\Omega$ by the symbol $*$, i.e. it will stand both for the usual and for the wedge product.

Let $h$ be the adjoint representation of $G$. Denote by $h_{\mu}^{v}$ its matrix elements: $\operatorname{Ad}_{g} e_{\mu}=h_{\mu}^{v}(g) e_{v}, g \in G$. Clearly, this implies that

$$
\Delta h_{\mu}^{v}=h_{\lambda}^{v} \otimes h_{\mu}^{\lambda}
$$

We use $\left(h_{\mu}^{v}\right)$ to introduce the linear mappings $\Delta_{r}: \Omega \rightarrow \Omega \otimes \mathscr{A}$ and $\Delta_{l}$ : $\Omega \rightarrow \mathscr{A} \otimes \Omega$, called the left and right coactions respectively.

\section{Definition 1.}

i) If $\phi \in \mathscr{A}$, then $\Delta_{r} \phi=\Delta_{l} \phi=\Delta \phi$; then

ii) let $\left\{\varepsilon^{\mu}\right\}$ be a basis of right-invariant forms in $\Omega^{1}$ corresponding to $\left\{e_{\mu}\right\}$,

$$
\begin{aligned}
& \Delta_{r} \varepsilon^{\mu}=\varepsilon^{\mu} \otimes I, \\
& \Delta_{l} \varepsilon^{\mu}=h_{v}^{\mu} \otimes \varepsilon^{v},
\end{aligned}
$$

where every matrix coefficient $h_{v}^{\mu}$ is regarded as an element of $\mathscr{A}$;

iii) if $\omega=\mu * v \in \Omega$, then

$$
\Delta_{r, l} \omega=\Delta_{r, l} \mu * \Delta_{r, l} \nu .
$$

With respect to $\Delta_{r, l}$ the space $\Omega$ is naturally endowed with the structure of a bicovariant bimodule [14]. Note that the definition of $\Delta_{r}\left(\Delta_{l}\right)$ is independent of the particular choice of a basis in $\Omega^{1}$. We use $\left\{\varepsilon^{\mu}\right\}$ as the most suitable for our treatment.

Now we introduce the following concept.

Definition 2. A bilinear operation $\{\}:, \Omega \otimes \Omega \rightarrow \Omega$ is called a bicovariant bracket if:

1) for any homogeneous elements $\omega, v, \rho \in \Omega$ it satisfies

i) the graded Leibnitz rule

$$
\{\omega * v, \rho\}=\omega *\{v, \rho\}+(-1)^{\operatorname{deg} v \operatorname{deg} \rho}\{\omega, \rho\} * v ;
$$

ii) the graded symmetry property

$$
\{\omega, v\}=-(-1)^{\operatorname{deg} \omega \operatorname{deg} v}\{v, \omega\}, \quad \operatorname{deg}\{\omega, v\}=(\operatorname{deg} \omega+\operatorname{deg} v) \bmod 2 ;
$$

2) on zero and first order differential forms the coactions $\Delta_{r, l}$ are the homomorphisms with respect to the bracket operation:

$$
\Delta_{r}\{\omega, v\}=\left\{\Delta_{r} \omega, \Delta_{r} v\right\}_{\Omega \otimes \mathscr{A}}, \quad \Delta_{l}\{\omega, v\}=\left\{\Delta_{l} \omega, \Delta_{l} v\right\}_{\mathscr{A} \otimes \Omega},
$$


where for any homogeneous elements $\mu, v, \omega, \rho \in \Omega$ :

$$
\{\mu \otimes v, \omega \otimes \rho\}_{\Omega \otimes \Omega}=\{\mu, \omega\} \otimes v * \rho+\mu * \omega \otimes\{v, \rho\} .
$$

If $1 . \mathrm{i})$ and 2) are fulfilled, then one can easily see that bicovariance conditions

$$
\Delta_{r, l}\{\omega, \rho\}=\left\{\Delta_{r, l} \omega, \Delta_{r, l} \rho\right\}_{\Omega \otimes \Omega}
$$

take place for any $\omega, \rho \in \Omega$. Moreover, a bicovariant bracket on $\Omega$ is completely defined as soon as it is defined on zero and first order forms. In the sequel the brackets $\left\{\varepsilon^{\mu}, \phi\right\}, \phi \in \mathscr{A}$ and $\left\{\varepsilon^{\mu}, \varepsilon^{\nu}\right\}$ will be refered to as the first and the second order brackets respectively.

Definition 3. A bicovariant bracket defines on $\Omega$ the structure of a graded Poisson Lie algebra if for any homogeneous elements $\omega, v, \rho \in \Omega$ it satisfies the Jacobi identity:

$$
(-1)^{\operatorname{deg} \omega \operatorname{deg} \rho}\{\{\omega, v\}, \rho\}+(-1)^{\operatorname{deg} \rho \operatorname{deg} v}\{\{\rho, \omega\}, v\}+(-1)^{\operatorname{deg} \omega \operatorname{deg} v}\{\{v, \rho\}, \omega\} .
$$

\section{Explicit Form of Bicovariant Bracket}

In this section supposing the existence of a bicovariant bracket on $\Omega$ we will derive the explicit form of its restriction on the subalgebra $\mathcal{N} \subset \Omega$ generated by the set $\left(h_{\alpha}^{\beta}, \varepsilon^{\mu}\right), \alpha, \beta, \mu=1, \ldots, \operatorname{dim} \mathscr{G}$. We use generators $h_{\alpha}^{\beta}$ and $\varepsilon^{\mu}$ as the most convenient to pose and solve the bicovariance condition (3.8) explicitly. In Sect. 6 we show how one can restore a bicovariant bracket on suitable generators of $\mathscr{A}$.

For the rest of the paper $G$ will be simple. Denote by $\hat{h}$ the adjoint representation of $\mathscr{G}$. In a basis $\left\{e_{\mu}\right\}$ of $\mathscr{G}$ with structure coefficients $c_{\mu \alpha}^{\beta}$ we have $\left(\hat{h}\left(e_{\mu}\right)\right)_{\alpha}^{\beta}=c_{\mu \alpha}^{\beta}$.

According to Definitions 1 and 2 a bicovariant bracket reduced on $\mathscr{A}$ defines on $G$ the structure of a Poisson Lie group. Thus, by virtue of (2.2) a bracket $\left\{h_{\alpha}^{\beta}, h_{\gamma}^{\delta}\right\}$ should have the form:

$$
\left\{h_{1}, h_{2}\right\}=\left[h_{1} h_{2}, r_{12}\right] \text {, }
$$

where $r_{12}$ is the $r$-matrix in representation $\hat{h}$. Since $h$ is taken to be the antihomomorphism $G \rightarrow$ Aut $\mathscr{G}$, the sign in the r.h.s. of (4.1) is opposite to (2.2).

Consider the second order bracket. Equation (3.8) shows that if $\omega$ and $\rho$ are right-invariant first order forms, then the bracket $\{\omega, \rho\}$ also defines a right-invariant element of $\Omega$. Therefore, taking into account the graded symmetry property (3.5) we have:

$$
\left\{\varepsilon^{\beta}, \varepsilon^{\delta}\right\}=\sum_{\text {even } k} \eta_{\mu_{1} \cdots \mu_{k}}^{\beta \delta} \varepsilon^{\mu_{1}} \wedge \cdots \wedge \varepsilon^{\mu_{k}}
$$

where for each $k$ a numerical tensor $\eta_{\mu_{1} \cdots \mu_{k}}^{\beta \delta}$ is symmetric in upper indices and absolutely antisymmetric in lower indices.

Further restrictions on $\eta$ 's are imposed by $\Delta_{l}$-covariance. Applying $\Delta_{l}$ to both sides of (4.2), we get

$$
\left\{h_{\alpha}^{\beta} \otimes \varepsilon^{\alpha}, h_{\gamma}^{\delta} \otimes \varepsilon^{\gamma}\right\}=h_{v_{1}}^{\mu_{1}} \cdots h_{v_{k}}^{\mu_{k}} \eta_{\mu_{1} \cdots \mu_{k}}^{\beta \delta} \otimes\left(\varepsilon^{\nu_{1}} \wedge \cdots \wedge \varepsilon^{v_{k}}\right) .
$$


Using Eq. (3.7) one can easily see that the last equation is equivalent to the set of relations:

$$
\text { for } k=2: \frac{1}{2}\left(\left\{h_{\alpha}^{\beta}, h_{\gamma}^{\delta}\right\}-\left\{h_{\gamma}^{\beta}, h_{\alpha}^{\delta}\right\}\right)+h_{\mu}^{\beta} h_{\nu}^{\delta} \eta_{\alpha \gamma}^{\mu \nu}=h_{\alpha}^{\mu} h_{\gamma}^{v} \eta_{\mu \nu}^{\beta \delta}
$$

and

$$
\text { for } k>2: \eta_{v_{1} \cdots v_{k}}^{\alpha \gamma} h_{\alpha}^{\beta} h_{\gamma}^{\delta}=h_{v_{1}}^{\mu_{1}} \cdots h_{v_{k}}^{\mu_{k}} \eta_{\mu_{1} \cdots \mu_{k}}^{\beta \delta} \text {. }
$$

With the help of the permutation matrix $P: P_{\alpha \beta}^{\mu \nu}=\delta_{\alpha}^{v} \delta_{\beta}^{\mu}$, Eq. (4.3) can be written as

$$
\frac{1}{2}\left(I-P_{12}\right)\left\{h_{1}, h_{2}\right\}=\left[h_{1} h_{2}, \eta_{12}\right] \text {. }
$$

By definition, put

$$
\varrho_{12}=\frac{1}{2}\left(I-P_{12}\right) r_{12}-\eta_{12} \text {. }
$$

Then, using (4.1), we see that Eq. (4.3) is equivalent to the following condition on $\varrho_{12}:\left[h_{1} h_{2}, \varrho_{12}\right]=0$.

We can use the nondegenerate Killing metric to raise and lower indices of $\eta$ 's. Clearly, for corresponding contravariant tensors Eq. (4.4) reads

$$
\eta^{\mu_{1} \cdots \mu_{k}} h_{\mu_{1}}^{\nu_{1}} \cdots h_{\mu_{k}}^{\nu_{k}}=\eta^{\nu_{1} \cdots \nu_{k}}
$$

i.e., $\eta^{\mu_{1} \cdots \mu_{k}}$ appears to be a $G$-invariant tensor.

Derivation of the first order bracket retraces the same steps. Let us sketch them briefly. The bracket $\left\{\varepsilon^{\beta}, h_{\gamma}^{\lambda}\right\}$ should be a differential form of odd range. Therefore,

$$
\left\{\varepsilon^{\beta}, h_{\gamma}^{\lambda}\right\}=\sum_{\text {odd } k}\left(\xi_{\mu_{1} \cdots \mu_{k}}^{\beta}\right)_{\gamma}^{\lambda} \varepsilon^{\mu_{1}} \wedge \cdots \wedge \varepsilon^{\mu_{k}}
$$

where this time for each set of indices an element $\left(\xi_{v_{1} \cdots v_{k}}^{\beta}\right)_{v}^{\lambda}$ belongs to $\mathscr{A}$.

Condition (3.8) of $\Delta_{r}$-covariance requires all $\xi$ in (4.8) to form a subset in $\mathscr{A}$ for which the comultiplication reduces to

$$
\Delta\left(\xi_{\mu_{1} \cdots \mu_{k}}^{\beta}\right)_{\gamma}^{\lambda}=\left(\xi_{\mu_{1} \cdots \mu_{k}}^{\beta}\right)_{v}^{\lambda} \otimes h_{\gamma}^{v} .
$$

Comparing the last equation with (3.1) one finds that it has a unique solution given by

$$
\left(\xi_{\mu_{1} \cdots \mu_{k}}^{\beta}\right)_{\gamma}^{\lambda}=h_{\gamma}^{\nu} \zeta_{\mu_{1} \cdots \mu_{k} v}^{\beta \lambda}
$$

Here for each $k \zeta_{\mu_{1} \cdots \mu_{k} v}^{\beta \lambda}$ is a numerical tensor.

Now it is not hard to verify that the requirement of $\Delta_{l}$-covariance yields the following first order bracket:

$$
\left\{\varepsilon^{\beta}, h_{\gamma}^{\lambda}\right\}=h_{\gamma}^{\delta} r_{\nu \delta}^{\beta \lambda} \varepsilon^{\nu}+\sum_{\text {odd } k} h_{\gamma}^{\delta} \zeta_{\mu_{1} \cdots \mu_{k} \delta}^{\beta \lambda} \varepsilon^{\mu_{1}} \wedge \cdots \wedge \varepsilon^{\mu_{k}},
$$

where tensors $\zeta_{\mu_{1} \cdots \mu_{k} \delta}^{\beta \lambda}$ obey the relation

$$
\zeta_{\mu_{1} \cdots \mu_{k} \delta}^{\beta \lambda} h_{\beta}^{\alpha} h_{\lambda}^{\gamma}=h_{\mu_{1}}^{v_{1}} \cdots h_{\mu_{1}}^{\nu_{1}} h_{\delta}^{\beta} \zeta_{v_{1} \cdots v_{k} \beta}^{\alpha \gamma} .
$$


Thus, we proved the

Theorem 1. For any simple connected Lie group the restriction of a bicovariant bracket on $\mathcal{N}$ is generated by the relations

$$
\begin{gathered}
\left\{h_{\mu}^{\gamma}, h_{\nu}^{\delta}\right\}=r_{\mu \nu}^{\alpha \beta} h_{\alpha}^{\gamma} h_{\beta}^{\delta}-h_{\mu}^{\alpha} h_{\nu}^{\beta} r_{\alpha \beta}^{\gamma \delta}, \\
\left\{\varepsilon^{\beta}, h_{\gamma}^{\lambda}\right\}=h_{\gamma}^{\delta} r_{\nu \delta}^{\beta \lambda} \varepsilon^{v}+\sum_{\text {odd } k} h_{\gamma}^{\delta} \zeta_{\mu_{1} \cdots \mu_{k} \delta}^{\beta \lambda} \varepsilon^{\mu_{1}} \wedge \cdots \wedge \varepsilon^{\mu_{k}}, \\
\left\{\varepsilon^{\beta}, \varepsilon^{\delta}\right\}=r_{\alpha \gamma}^{\beta \delta} \varepsilon^{\alpha} \wedge \varepsilon^{\gamma}+\sum_{\text {even } k} \eta_{\mu_{1} \cdots \mu_{k}}^{\beta \delta} \varepsilon^{\mu_{1}} \wedge \cdots \wedge \varepsilon^{\mu_{k}},
\end{gathered}
$$

where $r_{\alpha \gamma}^{\beta \delta}=r^{\mu v} c_{\mu \alpha}^{\beta} c_{v \gamma}^{\delta}$ is the r-matrix in representation $\hat{h}$ and corresponding contravariant tensors $\zeta, \eta$ are invariant with respect to the adjoint action. In addition $\eta_{\mu_{1} \cdots \mu_{k}}^{\beta \delta}$ is symmetric in upper indices and absolutely antisymmetric in lower indices, whereas $\zeta_{\mu_{1} \cdots \mu_{k} \delta}^{\beta \delta}$ is absolutely antisymmetric in $\mu_{1}, \ldots, \mu_{k}$.

Consider the tensors $\zeta_{\mu \nu}^{\alpha \beta}$ and $\eta_{\mu \nu}^{\alpha \beta}$ from the theorem. Their invariance means that $\zeta, \eta \in R\left(\mathscr{G}^{\otimes 2}, \mathscr{G}^{\otimes 2}\right)$, where $R\left(\mathscr{G}^{\otimes 2}, \mathscr{G}^{\otimes 2}\right)$ is the commuting algebra of $h \otimes h$. If we go over from the representation $h$ of a Lie group to the corresponding representation $\hat{h}$ of a Lie algebra the last observation can be expressed as

$$
[\hat{h}(X) \otimes I+I \otimes \hat{h}(X), Z]=0 \quad \text { for any } X \in \mathscr{G},
$$

where $Z$ is $\zeta$ or $\eta$.

If all tensors $\zeta$ and $\eta$ from Theorem 1 equal zero except $\zeta_{\mu \nu}^{\alpha \beta}$ and $\eta_{\mu \nu}^{\alpha \beta}$, then we say that the corresponding bicovariant bracket is homogeneous. Clearly, any homogeneous bicovariant bracket is characterized by the triple $(r, \zeta, \eta)$, where $\zeta, \eta$ belong to $R\left(\mathscr{G}^{\otimes 2}, \mathscr{G}^{\otimes 2}\right)$ and have the symmetry prescribed by Theorem 1 . In view of applications to bicovariant differential calculi on quantum groups in the sequel we confine ourselves to the study of homogeneous bicovariant brackets only.

\section{The Jacobi Identity}

In this section we examine the case when a homogeneous bicovariant bracket $(r, \zeta, \eta)$ defines on $\Omega$ the structure of a graded Poisson algebra.

It is readily seen that if $r$ is a solution of the mYBE, then the Jacobi identity for a homogeneous bicovariant bracket reduces to the following system of identities:

$$
\begin{gathered}
J_{1}=\left\{\left\{\varepsilon^{\mu}, h_{\gamma}^{\lambda}\right\}, h_{\omega}^{\kappa}\right\}-\left\{\left\{\varepsilon^{\mu}, h_{\omega}^{\kappa}\right\}, h_{\gamma}^{\lambda}\right\}-\left\{\varepsilon^{\mu},\left\{h_{\gamma}^{\lambda}, h_{\omega}^{\kappa}\right\}\right\}=0, \\
J_{2}=\left\{\left\{\varepsilon^{\mu}, \varepsilon^{\nu}\right\}, h_{\gamma}^{\lambda}\right\}-\left\{\left\{\varepsilon^{\mu}, h_{\gamma}^{\lambda}\right\}, \varepsilon^{\nu}\right\}+\left\{\varepsilon^{\mu},\left\{\varepsilon^{\nu}, h_{\gamma}^{\lambda}\right\}\right\}=0, \\
J_{3}=\left\{\left\{\varepsilon^{\beta}, \varepsilon^{\delta}\right\}, \varepsilon^{\nu}\right\}+\left\{\left\{\varepsilon^{\nu}, \varepsilon^{\beta}\right\}, \varepsilon^{\delta}\right\}+\left\{\left\{\varepsilon^{\delta}, \varepsilon^{\nu}\right\}, \varepsilon^{\beta}\right\}=0 .
\end{gathered}
$$

Solutions of (5.1)-(5.3) are described by the

Theorem 2. Let $r$ be a solution of the mYBE with a parameter $\alpha \neq 0$. A bicovariant homogeneous bracket $(r, \zeta, \eta)$ defines on $\Omega$ the structure of a graded Poisson 
algebra if the following conditions hold:

i)

$$
\left[\zeta_{13}, \zeta_{23}\right]=\alpha\left[K_{13}, K_{23}\right]
$$

ii)

$$
\alpha\left[K_{13}, K_{23}\right]_{\alpha \mu \lambda}^{\beta \delta v}-2 \sum_{(\beta, \delta, v)(\alpha, \mu, \lambda)} \eta_{\mu \lambda}^{\gamma v} \eta_{\alpha \gamma}^{\beta \delta}=0 .
$$

Moreover, Eqs. (5.1) and (5.2) are fulfilled regardless of ii).

Note that in Eq. (5.5) the sum is taken both over the cyclic permutations of $(\beta, \delta, v)$ and over the cyclic permutations of $(\alpha, \mu, \lambda)$.

Proof. To simplify the calculations we put $\left\{\varepsilon^{\mu}, h_{\gamma}^{\lambda}\right\}=h_{\gamma}^{\delta} \sigma_{v \delta}^{\mu \lambda} \varepsilon^{\nu}$ and $\left\{\varepsilon^{\mu}, \varepsilon^{\nu}\right\}=$ $A_{\alpha \beta}^{\mu \nu} \varepsilon^{\alpha} \wedge \varepsilon^{\beta}$, where $\sigma_{v \delta}^{\mu \lambda}=r_{v \delta}^{\mu \lambda}+\zeta_{v \delta}^{\mu \lambda}$ and $A_{\alpha \beta}^{\mu v}=r_{\alpha \beta}^{\mu v}+\eta_{\alpha \beta}^{\mu \nu}$.

We start with (5.1). By straightforward calculations, one obtains

$$
J_{1}=-\varepsilon^{v} h_{\omega}^{\alpha} h_{\gamma}^{\beta} M_{v \alpha \beta}^{\mu \kappa \lambda},
$$

where $M$ is of the form

$$
M=\left[\sigma_{12}, \sigma_{13}+\sigma_{23}\right]+\left[\sigma_{13}, \sigma_{23}\right] .
$$

Now substituting $\sigma$ in (5.6) and having in mind that $\zeta \in R\left(\mathscr{G}^{\otimes 2}, \mathscr{G}^{\otimes 2}\right.$ ) (see Eq. (4.16)), we get $M=[[r, r]]+\left[\zeta_{13}, \zeta_{23}\right]$. Thus, if $\zeta$ obeys Eq. (5.4) identity (5.1) is fulfilled due to the mYBE.

The next identity involves two $\varepsilon$ and one $h$ generators. This time we find

$$
J_{2}=-\frac{1}{2} \varepsilon^{\alpha} \wedge \varepsilon^{\beta} h_{\gamma}^{\kappa}\left(L_{\alpha \beta \kappa}^{\mu \nu \lambda}-L_{\beta \alpha \kappa}^{\mu \nu \lambda}\right)
$$

where $L$ is of the form

$$
L=\left[A_{12}, \sigma_{13}+\sigma_{23}\right]+\left[\sigma_{13}, \sigma_{23}\right] .
$$

Since both $\zeta$ and $\eta$ satisfies Eq. (4.16), we obtain that $L=-\left[\eta_{12}, \zeta_{13}+\zeta_{23}\right]=0$. Hence, under condition (5.4) identity (5.2) is satisfied automatically, i.e., it does not contain any new restrictions on $\zeta$ and $\eta$.

Substituting the explicit form of the bracket $\left\{\varepsilon^{\mu}, \varepsilon^{\nu}\right\}$ in the 1.h.s. of identity (5.3) one gets

$$
J_{3}=\left\{\left(r_{\alpha \gamma}^{\beta \delta}+\eta_{\alpha \gamma}^{\beta \delta}\right) \varepsilon^{\alpha} \wedge \varepsilon^{\gamma}, \varepsilon^{\nu}\right\}+\text { c.p. }
$$

One more substitution of $\left\{\varepsilon^{\mu}, \varepsilon^{\nu}\right\}$ into (5.8) results in

$$
\begin{aligned}
J_{3}=\sum_{(\beta, \delta, v)}\left\{\left\{\varepsilon^{\beta}, \varepsilon^{\delta}\right\}, \varepsilon^{v}\right\}= & \sum_{(\beta, \delta, v)}\left(r_{\alpha \gamma}^{\beta \delta} r_{\mu \lambda}^{\gamma v}-r_{\gamma \alpha}^{\beta \delta} r_{\mu \lambda}^{\gamma v}\right) \varepsilon^{\alpha} \wedge \varepsilon^{\mu} \wedge \varepsilon^{\lambda} \\
& +\sum_{(\beta, \delta, v)}\left(\left(\eta_{\alpha \gamma}^{\beta \delta}-\eta_{\gamma \alpha}^{\beta \delta}\right) r_{\mu \lambda}^{\gamma v}+\left(r_{\alpha \gamma}^{\beta \delta}-r_{\gamma \alpha}^{\beta \delta}\right) \eta_{\mu \lambda}^{\gamma v}\right) \varepsilon^{\alpha} \wedge \varepsilon^{\mu} \wedge \varepsilon^{\lambda} \\
& -\sum_{(\beta, \delta, v)}\left(\left(\eta_{\gamma \alpha}^{\beta \delta}-\eta_{\alpha \gamma}^{\beta \delta}\right) \eta_{\mu \lambda}^{\gamma v}\right) \varepsilon^{\alpha} \wedge \varepsilon^{\mu} \wedge \varepsilon^{\lambda}
\end{aligned}
$$


The further analysis of (5.9) will be done in two steps.

1. As the first step we consider only the first sum coming in (5.9). Performing the cyclic permutations it is easy to find that

$$
\sum_{(\beta, \delta, v)}\left(r_{\alpha \gamma}^{\beta \delta} r_{\mu \lambda}^{\gamma v}-r_{\gamma \alpha}^{\beta \delta} r_{\mu \lambda}^{\gamma v}\right) \varepsilon^{\alpha} \wedge \varepsilon^{\mu} \wedge \varepsilon^{\lambda}=-[[r, r]]_{\alpha \mu \lambda}^{\beta \delta v} \varepsilon^{\alpha} \wedge \varepsilon^{\mu} \wedge \varepsilon^{\lambda}
$$

i.e., it reduces to the 1.h.s. of the mYBE.

2. As the second step let us consider the contribution to $J_{3}$ delivered by the second sum of (5.9). After performing the cyclic permutations this sum can be written as

$$
\begin{aligned}
& \left(\eta_{\mu \alpha}^{\gamma \beta} r_{\gamma \lambda}^{\delta v}-r_{\mu \lambda}^{\gamma v} \eta_{\gamma \alpha}^{\delta \beta}+\eta_{\mu \alpha}^{\delta \gamma} r_{\gamma \lambda}^{\beta v}-r_{\alpha \lambda}^{\gamma v} \eta_{\mu \gamma}^{\delta \beta}\right) \varepsilon^{\alpha} \wedge \varepsilon^{\mu} \wedge \varepsilon^{\lambda} \\
& \quad-\left(\eta_{\mu \lambda}^{\gamma v} r_{\gamma \alpha}^{\beta \delta}-r_{\mu \gamma}^{\alpha \delta} \eta_{\gamma \lambda}^{\beta v}+\eta_{\mu \lambda}^{\beta \gamma} r_{\gamma \alpha}^{\nu \delta}-r_{\lambda \alpha}^{\gamma \delta} \eta_{\mu \gamma}^{\beta v}\right) \varepsilon^{\alpha} \wedge \varepsilon^{\mu} \wedge \varepsilon^{\lambda} \\
& \quad+\left(\eta_{\mu \alpha}^{\gamma \delta} r_{\gamma \alpha}^{\nu \beta}-r_{\mu \lambda}^{\gamma \beta} \eta_{\gamma \alpha}^{v \delta}+\eta_{\mu \alpha}^{\nu \gamma} r_{\gamma \lambda}^{\delta \beta}-r_{\alpha \lambda}^{\gamma \beta} \eta_{\mu \gamma}^{v \delta}\right) \varepsilon^{\alpha} \wedge \varepsilon^{\mu} \wedge \varepsilon^{\lambda} .
\end{aligned}
$$

Now using the fact that $r$-matrix is taken in representation $\hat{h}$ expression (5.11) can be written as

$$
\begin{aligned}
& r^{\gamma \rho}\left(\left[\eta, \hat{h}\left(e_{\gamma}\right) \otimes I+I \otimes \hat{h}\left(e_{\gamma}\right)\right]\right)_{\mu \alpha}^{\delta \beta} \hat{h}\left(e_{\rho}\right)_{\lambda}^{\nu} \varepsilon^{\alpha} \wedge \varepsilon^{\mu} \wedge \varepsilon^{\lambda} \\
& \quad+r^{\gamma \rho}\left(\left[\hat{h}\left(e_{\gamma}\right) \otimes I+I \otimes \hat{h}\left(e_{\gamma}\right), \eta\right]\right)_{\mu \lambda}^{\beta \nu} \hat{h}\left(e_{\rho}\right)_{\alpha}^{\delta} \varepsilon^{\alpha} \wedge \varepsilon^{\mu} \wedge \varepsilon^{\lambda} \\
& \quad+r^{\gamma \rho}\left(\left[\eta, \hat{h}\left(e_{\gamma}\right) \otimes I+I \otimes \hat{h}\left(e_{\gamma}\right)\right]\right)_{\mu \alpha}^{\nu \delta} \hat{h}\left(e_{\rho}\right)_{\lambda}^{\beta} \varepsilon^{\alpha} \wedge \varepsilon^{\mu} \wedge \varepsilon^{\lambda} .
\end{aligned}
$$

Since $\eta$ is invariant under the adjoint action of $\mathscr{G}$ each summand coming in (5.12) is equal to zero and, therefore, the sum under consideration does not contribute to the 1.h.s. of the Jacobi identity.

\section{Fundamental Poisson Bracket}

The aim of this section is to find solutions of Eq. (5.5) for simple complex Lie groups corresponding to classical series. This will be achieved by introducing the fundamental Poisson bracket. The main result to be proven here is the

Theorem 3. Let $G$ be a simple Lie group corresponding to one of $A_{n-1}, B_{n}, C_{n}$, $D_{n}$ series.

i) If $G$ is of type $A_{n-1}, n>2$ then $\Omega$ admits four different Poisson Lie structures defined by homogeneous brackets;

ii) If $G$ is $A_{1}$, then $\Omega$ admits two different Poisson Lie structures defined by homogeneous brackets;

iii) if $G$ is one of types $B_{n}(n \geqq 2), C_{n}(n \geqq 3)$ or $D_{n}(n \geqq 4)$, then the Poisson Lie structure defined by homogeneous brackets does not exist.

Denote by $\theta=\varepsilon^{\mu} e_{\mu}=\left(\theta_{i}^{j}\right)$ the Lie-valued right-invariant canonical one-form on $G$. Let $G$ be realized as a matrix group, i.e., $G \ni g=\left(t_{i}^{j}\right)_{i, j=1}^{N}$ in the space $V$. 
Consider $T=\left(t_{i}^{j}\right)$ as the fundamental holomorphic representation of $G$. Note that the set $\left(\theta_{i}^{j}, t_{i}^{j}\right)$ generates $\Omega$.

Now by using Eqs. (4.14) and (4.15) we define on $\Omega$ the structure of a graded Poisson algebra in terms of generators $\left(\theta_{i}^{j}, t_{i}^{j}\right)$. Following [1] we will refer to the corresponding brackets between these generators as fundamental. Note that entries of $\theta$ as well as entries of $T$ are not independent so there will be some relations between brackets involving different generators.

According to Sect. 2 the bracket $\left\{t_{i}^{j}, t_{k}^{l}\right\}$ coincides with (2.2), where $\tau$ is the identical representation of $\mathscr{G}$ ( $\mathscr{G}$ is a matrix algebra).

Evidently, we can put

$$
\left\{\theta_{i}^{j}, \theta_{k}^{l}\right\}=\left\{\varepsilon^{\mu}, \varepsilon^{v}\right\}\left(e_{\mu}\right)_{i}^{j}\left(e_{v}\right)_{k}^{l} .
$$

Substituting (4.15) in (6.1), we get

$$
\left\{\theta_{i}^{j}, \theta_{k}^{l}\right\}=\left(r_{\alpha \beta}^{\mu \nu}+\eta_{\alpha \beta}^{\mu v}\right)\left(e_{\mu}\right)_{i}^{j}\left(e_{v}\right)_{k}^{l} \varepsilon^{\alpha} \wedge \varepsilon^{\beta}
$$

The first summand in the r.h.s. of (6.2) can be written as

$$
\begin{aligned}
r^{\gamma \delta} c_{\gamma \alpha}^{\mu} c_{\delta \beta}^{v}\left(e_{\mu}\right)_{i}^{j}\left(e_{v}\right)_{k}^{l} \varepsilon^{\alpha} \wedge \varepsilon^{\beta} & =r^{\gamma \delta}\left[e_{\gamma}, e_{\alpha}\right]_{i}^{j}\left[e_{\delta}, e_{\beta}\right]_{k}^{l} \varepsilon^{\alpha} \wedge \varepsilon^{\beta} \\
& =r^{\gamma \delta}\left[e_{\gamma}, \theta\right]_{i}^{j} \wedge\left[e_{\delta}, \theta\right]_{k}^{l} .
\end{aligned}
$$

In the second summand we raise indices of tensor $\eta$ with the help of the Killing metric $g_{\mu \nu}=\operatorname{tr}\left(e_{\mu} e_{\nu}\right)$ :

$$
\eta^{\mu \nu \gamma \delta} g_{\gamma \alpha} g_{\delta \beta}\left(e_{\mu}\right)_{i}^{j}\left(e_{v}\right)_{k}^{l} \varepsilon^{\alpha} \wedge \varepsilon^{\beta}=\eta^{\mu \nu \gamma \delta}\left(e_{\mu}\right)_{i}^{j}\left(e_{v}\right)_{k}^{l} \operatorname{tr}\left(e_{\gamma} \theta\right) \wedge \operatorname{tr}\left(e_{\delta} \theta\right) .
$$

Denote by $\eta_{1234} \in \mathscr{G}^{\otimes 4}$ (here the subscripts label the corresponding factors in $\mathscr{G}^{\otimes 4}$ ) a tensor:

$$
\eta_{i k n p}^{j l m s}=\eta^{\mu v \gamma \delta}\left(e_{\mu}\right)_{i}^{j}\left(e_{v}\right)_{k}^{l}\left(e_{\gamma}\right)_{n}^{m}\left(e_{\delta}\right)_{p}^{s} .
$$

Note that by Theorem $1 \eta_{1234}$ is an element of $S^{2} \mathscr{G} \otimes \wedge^{2} \mathscr{G}$, where $S^{2} \mathscr{G}$ stands for the symmetric part of $\mathscr{G}^{\otimes 2}$.

Combining (6.2)-(6.4) and adopting tensor notation introduced in Sect. 2, we get for (6.1),

$$
\left\{\theta_{1}, \theta_{2}\right\}=\left\{\theta_{1},\left[\theta_{2}, r_{12}\right]\right\}+\operatorname{tr}_{34}\left(\eta_{1234} \theta_{3} \theta_{4}\right),
$$

where trace with subscript stands for the matrix trace applied to corresponding factors in $\mathscr{G}^{\otimes 4}$. In the r.h.s. of Eq. (6.6) we use square brackets (braces) for the commutator (anticommutator) in a matrix algebra. To simplify the notation we omit here and below the sign of the wedge product of differential forms keeping in mind that $\theta_{i}^{j} \theta_{k}^{l}=-\theta_{k}^{l} \theta_{i}^{j}$.

To make the picture complete we write out the brackets $\left\{\theta_{i}^{j}, t_{k}^{l}\right\}$. According to [2] for simple $\mathscr{G}$ Eq. (4.14) has only two solutions $\zeta= \pm \sqrt{\alpha} K$, where $K=g^{\mu v} e_{\mu} \otimes$ $e_{\nu}$. Multiplying both sides of (4.14) by $\left(e_{\mu}\right)_{i}^{j}\left(e_{\lambda}\right)_{k}^{l}$ and using the same arguments as above, we get

$$
\left\{\theta_{i}^{j},\left(T e_{\gamma} S(T)\right)_{k}^{l}\right\}_{ \pm}=\left(r^{\mu v} \pm \sqrt{\alpha} g^{\mu v}\right)\left[e_{\mu}, \theta\right]_{i}^{j}\left[e_{v}, T e_{\gamma} S(T)\right]_{k}^{l} .
$$

Here the subscript \pm on the l.h.s. indicates the choice of $\zeta$ and $S(T)=T^{-1}$ stands for the antipod of $T$. 
The bracket on the 1.h.s. of (6.7) satisfies the obvious identity: $\left\{\theta_{i}^{j}, T e_{\gamma} S(T)\right\}_{ \pm}$ $=T\left(\left[S(T)\left\{\theta_{i}^{j}, T\right\}_{ \pm}, e_{\gamma}\right]\right) S(T)$, where for each $i$ and $j[$, ] means the commutator of the matrices: $S(T)\left\{\theta_{i}^{j}, T\right\}_{ \pm}$and $e_{\gamma}$. Therefore, for (6.7) we have

$$
\left[S(T)\left\{\theta_{i}^{j}, T\right\}_{ \pm}, e_{\gamma}\right]_{k}^{l}=\left(r^{\mu \nu} \pm \sqrt{\alpha} g^{\mu \nu}\right)\left[e_{\mu}, \theta\right]_{i}^{j}\left[S(T) e_{\nu} T, e_{\gamma}\right]_{k}^{l} .
$$

Since $\hat{h}$ is irreducible the last equation is solved elementary and we get two $( \pm)$ brackets

$$
\left\{\theta_{1}, T_{2}\right\}_{ \pm}=\left[r_{12} \pm \sqrt{\alpha} K_{12}, \theta_{1}\right] T_{2} .
$$

Hence, relations (2.2), (6.6) and (6.9) are the defining relations of a bicovariant bracket on $\Omega$.

The next lemma is an analog of Theorem 2 for the fundamental Poisson bracket.

Lemma 1. Suppose $r \in \wedge^{2} \mathscr{G}$ is a solution of the $m Y B E$ with a parameter $\alpha \neq 0$; then the bicovariant bracket given by Eqs. (2.2),(6.6), and (6.9), defines the fundamental Poisson bracket iff there exists an invariant element $\eta_{1234} \in S^{2} \mathscr{G} \otimes \wedge^{2} \mathscr{G}$ such that the following relation is satisfied:

$$
\alpha\left[\theta_{1},\left\{\theta_{2},\left[\theta_{3},\left[K_{13}, K_{23}\right]\right]\right\}\right]-2 \operatorname{tr}_{456}\left(\Im_{123456} \theta_{4} \theta_{5} \theta_{6}\right)=0,
$$

where

$$
\Im_{123456}=\sum_{\text {c.p. }} \operatorname{tr}_{\gamma}\left(\eta_{12 \gamma 6} \eta_{3 \gamma 45}\right) .
$$

Proof. According to Theorem 2 what we need is to check that the identity $\sum_{\text {c.p. }(123)}\left\{\left\{\theta_{1}, \theta_{2}\right\}, \theta_{3}\right\}=0$ reduces to Eq. (6.10). By construction of the fundamental bracket this statement follows directly from Eq. (5.5).

Proof of Theorem 3. By Lemma $1 \Omega$ admits the graded Poisson Lie structure defined by a homogeneous bracket if there exists a $G$-invariant tensor $\eta$ such that the corresponding $\eta_{1234}$ satisfies (6.10). Our strategy will be the following. First we describe explicitly all invariant tensors in $S^{2} \mathscr{G} \otimes \wedge^{2} \mathscr{G}$. The second step will be to check which of them obeys (6.10).

For later convenience we introduce the generators $\left\{f_{i}^{j}\right\}$ corresponding to oneparameter subgroups of $G$ :

$$
\left(f_{i}^{j}\right)_{k}^{l}=\delta_{i}^{l} \delta_{k}^{j}-\frac{1}{N} \delta_{i}^{j} \delta_{k}^{l} \quad \text { for } A_{n-1} \text { series }
$$

and

$$
\left(f_{i}^{j}\right)_{k}^{l}=\delta_{i}^{l} \delta_{k}^{j}-\varepsilon C_{i k} C^{j l} \quad \text { for } B_{n}, C_{n} \text { and } D_{n} \text { series, }
$$

where $\varepsilon=1$ for $B_{n}, D_{n}$ and $\varepsilon=-1$ for $C_{n}$.

Having in mind that $G$ is realized as the matrix group in the space $V$ we consider a tensor representation $\pi$ of $G$ in $W=V^{\otimes 4}$.

Lemma 2. If $\eta^{\mu \nu \alpha \beta}$ is a G-invariant tensor, then $\eta_{1234} \in \mathscr{G}^{\otimes 4}$ belongs to the commuting algebra $R(W, W)$ of $W$.

The proof follows easily from Definition (6.5).

The first and the second statements of Theorem 3 deal with $A_{n-1}$ series and the third one with $B_{n}, C_{n}$ and $D_{n}$. According to this we divide the proof of the theorem in two parts.

1) The next well-known lemma describes $R(W, W)$ for $G$ of type $A_{n-1}$. 
Lemma 3 [27]. Let $G$ be of type $A_{n-1}$, then $R(W, W)=\Re$, where $\Re$ is the group algebra of the permutation group $S_{4}$.

Thus, the general form of $\eta_{1234} \in R(W, W)$ is

$$
\eta_{i k m p}^{j l n s}=\sum_{\sigma \in S_{4}} \lambda(\sigma) \delta_{\sigma(i)}^{j} \delta_{\sigma(k)}^{l} \delta_{\sigma(m)}^{n} \delta_{\sigma(p)}^{s}, \quad \lambda(\sigma) \in \mathbf{C}
$$

Here the r.h.s. realizes the representation of the group algebra of $S_{4}$ in $W$.

Lemma 4. If $G$ is of type $A_{n-1}$, then in the space $S^{2} \mathscr{G} \otimes \wedge^{2} \mathscr{G}$ there is a unique (up to a multiplicative constant) element $\eta_{1234} \in R(W, W)$ given by

$$
\eta_{i k m s}^{j l n p}=\operatorname{tr}\left(\left(f_{i}^{j} f_{k}^{l}+f_{k}^{l} f_{i}^{j}\right)\left[f_{m}^{n}, f_{s}^{p}\right]\right) .
$$

Proof. Consider Eq. (6.13). The requirement on $\eta_{1234}$ to be an element of $S^{2} \mathscr{G} \otimes \wedge^{2} \mathscr{G}$ defines it uniquely (up to a multiplicative constant):

$$
\begin{aligned}
\eta_{i k m n}^{j l s p}= & \delta_{k}^{j} \delta_{i}^{s} \delta_{m}^{p} \delta_{n}^{l}+\delta_{i}^{l} \delta_{k}^{s} \delta_{m}^{p} \delta_{n}^{j}-\delta_{i}^{p} \delta_{k}^{j} \delta_{n}^{s} \delta_{m}^{l}-\delta_{i}^{l} \delta_{m}^{j} \delta_{k}^{p} \delta_{s}^{n} \\
& -\frac{2}{N} \delta_{k}^{l} \delta_{i}^{s} \delta_{m}^{p} \delta_{n}^{j}-\frac{2}{N} \delta_{i}^{j} \delta_{k}^{s} \delta_{m}^{p} \delta_{n}^{l}+\frac{2}{N} \delta_{i}^{p} \delta_{n}^{s} \delta_{k}^{l} \delta_{m}^{j}+\frac{2}{N} \delta_{l}^{j} \delta_{m}^{l} \delta_{k}^{p} \delta_{n}^{s}
\end{aligned}
$$

Such a tensor $\eta_{1234}$ being written via generators $\left\{f_{i}^{j}\right\}$ for $A_{n-1}$ acquires the form (6.14). Note that in this form the desired symmetry of $\eta_{1234}$ is obvious.

Substituting the explicit form (6.15) of $\eta_{1234}$ multiplied by an arbitrary constant, say $\beta$, in (6.10) it is not hard to prove by direct calculations that $(6.10)$ is fulfilled only for two values of $\beta: \beta= \pm \sqrt{\alpha}$. To complete the proof of 1) we write down the corresponding bracket $\left\{\theta_{1}, \theta_{2}\right\}_{ \pm}$labeled by the sign + or - :

$$
\left\{\theta_{1}, \theta_{2}\right\}_{ \pm}=\left\{\theta_{1}\left[\theta_{2}, r\right]\right\} \pm \sqrt{\alpha}\left(\theta_{1} P \theta_{2}+\theta_{2} P \theta_{1}-\frac{2}{N} \theta_{1} \theta_{1}-\frac{2}{N} \theta_{2} \theta_{2}\right)
$$

where $P$ is a permutation matrix: $P_{i j}^{k l}=\delta_{i}^{k} \delta_{j}^{l}$.

If $N=2$, then the sum in parentheses equals zero. Therefore for $\operatorname{SL}(2, \mathbf{C})$ the bracket $\left\{\theta_{1}, \theta_{2}\right\}$ is unique. Combining it with one of (6.9) we get the graded Poisson Lie structure on $\operatorname{SL}(2, \mathbf{C})$.

It remains to note that when $N>2$ fundamental Poisson brackets (2.2), (6.9), and (6.16) define four different (by Theorem 2 four choices of the signs \pm in (6.9) and (6.16) are admissible) graded Poisson Lie structures on $\Omega$.

2) The analog of Lemma 2 for $G$ belonging to one of types $B_{n}, C_{n}$ or $D_{n}$ is the following

Lemma 5. Let $G$ be one of types $B_{n}(n \geqq 2), C_{n}(n \geqq 3)$ or $D_{n}(n>4)$, then $a$ general form of $\eta_{1234} \in R(W, W)$ is

$$
\begin{aligned}
\eta_{i k m p}^{j l n s}= & \sum_{\sigma \in S_{4}} \lambda_{1}(\sigma) \delta_{\sigma(i)}^{j} \delta_{\sigma(k)}^{l} \delta_{\sigma(m)}^{n} \delta_{\sigma(p)}^{s}+\sum_{\sigma, \varepsilon \in S_{4}} \lambda_{2}(\sigma, \varepsilon) C_{\sigma(i) \sigma(k)} C^{\varepsilon(j) \varepsilon(l)} \delta_{\sigma(m)}^{\sigma(n)} \delta_{\sigma(p)}^{\sigma(s)} \\
& +\sum_{\sigma, \varepsilon \in S_{4}} \lambda_{3}(\sigma, \varepsilon) C_{\sigma(i) \sigma(k)} C_{\sigma(m) \sigma(p)} C^{\varepsilon(J) \varepsilon(l)} C^{\varepsilon(n) \varepsilon(p)}
\end{aligned}
$$

where all the coefficients $\lambda_{i} \in \mathbf{C}, i=1,2,3$. 
The proof is analogous to the case of $A_{n-1}$ and will be omitted. We only stress that the presence of the second and the third sum in (6.17) is due to the metric $C$, which gives rise to the contraction operation commuting with the group action in $W$. Constraints on $n$ indicated in the lemma become clear due to the remark below.

Remark. Suppose $G=S O(N, \mathbf{C})$; then there exists the absolutely antisymmetric $G$ invariant tensor $\varepsilon^{i_{1} \cdots i_{N}}$. One may use it to construct new elements from $R(W, W)$. If $N>8$, then there are no elements in $R(W, W)$ generated by $\varepsilon^{i_{1} \cdots i_{N}}$. If $N=8$, then elements of the type

$$
\eta_{i k m p}^{j l n s}=\varepsilon^{j l n s i_{1} i_{2} i_{3} i_{4}} C_{i i_{1}} C_{k i_{2}} C_{m i_{3}} C_{p i_{4}}
$$

belong to $R(W, W)$. However, using this $\eta$ it is impossible to form a tensor with symmetry properties required by Theorem 2 . It is also readily seen that the absolutely antisymmetric tensor for $S O(5, \mathrm{C})$ and $S O(7, \mathrm{C})$ does not contribute to $R(W, W)$.

Let $G$ be one of $S O(3, \mathrm{C}), S O(4, \mathrm{C})$ or $S O(6, \mathbf{C})$, then the corresponding absolutely antisymmetric tensors generate new elements of $R(W, W)$. For example, in the case of $S O(6, \mathrm{C})$ there exists the following tensor with the desired symmetry:

$$
\eta_{i k m p}^{j l n s}=\varepsilon_{i m p}^{j l n} \delta_{k}^{s}+\varepsilon_{k m p}^{j l n} \delta_{i}^{s}-\varepsilon_{i m p}^{j l s} \delta_{k}^{n}-\varepsilon_{k m p}^{j l s} \delta_{i}^{n},
$$

where $\varepsilon_{i m p}^{j l n}=\varepsilon^{j l n i_{1} i_{2} i_{3}} C_{i i_{1}} C_{m i_{2}} C_{p i_{3}}$. However, by virtue of classical isomorphisms of Lie algebras:

$$
\begin{aligned}
& \operatorname{so}(3, \mathbf{C}) \sim \operatorname{sl}(2, \mathbf{C}) \sim \operatorname{sp}(2, \mathbf{C}), \\
& \operatorname{so}(4, \mathbf{C}) \sim \operatorname{sl}(2, \mathbf{C}) \oplus \operatorname{sl}(2, \mathbf{C}), \\
& \operatorname{so}(6, \mathbf{C}) \sim \operatorname{sl}(4, \mathbf{C})
\end{aligned}
$$

the study of the commuting algebra for corresponding $G$ reduces to the previous case of the $A_{n-1}$ series.

Lemma 6. If $G$ is one of types $B_{n}(n \geqq 2), C_{n}(n \geqq 3)$ or $D_{n}(n \geqq 4)$, then in the space $S^{2} \mathscr{G} \otimes \wedge^{2} \mathscr{G}$ there is no nontrivial element $\eta_{1234} \in R(W, W)$.

Proof. First we find the values of $\lambda_{i}$ for which tensor $\eta_{1234}$ becomes an element of $\mathscr{G}^{\otimes 4}$. For this purpose we project the general form (6.17) on the space $\mathscr{G}^{\otimes 4}$. Let $\Theta$ be an operator in Mat $(N, \mathbf{C})^{\otimes 4}$ with matrix coefficients:

$$
(\Theta)_{i_{1} \cdots i_{4} k_{1} \cdots k_{4}}^{j_{1} \cdots j_{4} l_{1} \cdots l_{4}}=\left(f_{i_{1}}^{j_{1}}\right)_{k_{1}}^{l_{1}} \cdots\left(f_{i_{4}}^{j_{4}}\right)_{k_{4}}^{l_{4}} .
$$

It is easy to see that if $\eta \in$ Mat $(N, \mathbf{C})^{\otimes 4}$, then $\Theta \eta \in \mathscr{G}^{\otimes 4}$ and this projection commutes with the group action.

The set of generators $\left\{f_{i}^{j}\right\}$ form a tensor $f_{12}$ with elements $f_{i k}^{j l}=\left(f_{i}^{j}\right)_{k}^{l}$. Then Definition (6.12) implies that $f_{12} \in \mathscr{G}^{\otimes 2}$, i.e. for each $i=1,2$ the following relation holds:

$$
f_{12}^{t_{l}}=-C_{i} f_{12} C_{i}^{-1}
$$

Here $t_{i}$ stands for the matrix transposition in $i$ 's factor of $\mathscr{G}^{\otimes 2}$ and $C_{i}$ is the matrix $C$ acting in the space $i$ of $\mathscr{G}^{\otimes 2}$. 
Acting on (6.17) with $\Theta$ and using (6.18) we obtain the most general form of $\hat{\eta}_{1234} \in R(W, W)$ lying in the space $\mathscr{G}^{\otimes 4}$ :

$$
\begin{aligned}
\hat{\eta}_{i k m p}^{j l n s}= & (\Theta)_{i k m p j_{1} j_{2} j_{3} j_{4}}^{j l n i_{1} i_{i} i_{3} i_{4}} \eta_{i_{1} i_{2} i_{3} i_{4}}^{j_{1} j_{2} j_{3} j_{4}} \\
= & \sum_{\sigma \in S_{4}} \mu_{1}(\sigma) \operatorname{tr}\left(\sigma\left(f_{i}^{j}\right) \sigma\left(f_{k}^{l}\right)\right) \operatorname{tr}\left(\sigma\left(f_{m}^{n}\right) \sigma\left(f_{s}^{p}\right)\right) \\
& +\sum_{\sigma \in S_{4}} \mu_{2}(\sigma) \operatorname{tr}\left(\sigma\left(f_{i}^{j}\right) \sigma\left(f_{k}^{l}\right) \sigma\left(f_{m}^{n}\right) \sigma\left(f_{s}^{p}\right)\right),
\end{aligned}
$$

where for each permutation $\sigma$ the numerical coefficients $\mu_{1}(\sigma), \mu_{2}(\sigma)$ are some combinations of $\lambda_{i}$.

Now to obtain an element $\tilde{\eta}_{1234} \in S^{2} \mathscr{G} \otimes \wedge^{2} \mathscr{G}$ we apply to factors 1 and 2 of $\hat{\eta}_{1234}$ the symmetrization and to factors 3 and 4 the antisymmetrization:

$$
\tilde{\eta}_{1234}=\hat{\eta}_{1234}+\hat{\eta}_{2134}-\hat{\eta}_{1243}-\hat{\eta}_{2143} \text {. }
$$

This yields a unique (up to a multiplicative constant) tensor:

$$
\tilde{\eta}_{i k m s}^{j l n p}=\operatorname{tr}\left(\left(f_{i}^{j} f_{k}^{l}+f_{k}^{l} f_{i}^{j}\right)\left[f_{m}^{n}, f_{s}^{p}\right]\right),
$$

which is of the same form as for $A_{n-1}$ series (cf. (6.14)). However, $\tilde{\eta}_{1234}=0$. Indeed, the generators $f_{i}^{j}$ have the following commutation relations:

$$
\left[f_{i}^{j}, f_{k}^{l}\right]=\frac{1}{2} D_{i k m}^{j l n} f_{n}^{m}
$$

where

$$
\begin{aligned}
D_{i k m}^{j l n}= & \delta_{i}^{l} \delta_{k}^{n} \delta_{m}^{j}-\delta_{i}^{n} \delta_{k}^{j} \delta_{m}^{l}+C_{i k} C^{n j} \delta_{m}^{l}+C_{k m} C^{j l} \delta_{i}^{n}+C_{m k} C^{j n} \delta_{i}^{l} \\
& +C_{m i} C^{l n} \delta_{k}^{j}-C_{i k} C^{l n} \delta_{m}^{j}-C_{m i} C^{j l} \delta_{k}^{n}
\end{aligned}
$$

By using the explicit form of generators $f_{i}^{j}$ one can also check the useful identity:

$$
D_{i k m}^{j l n}=\operatorname{tr}\left(f_{i}^{j} f_{k}^{l} f_{m}^{n}\right) .
$$

Now it is not hard to prove that $D \in \wedge^{3} \mathscr{G}$ is $G$-invariant. (Recall that for simple $\mathscr{G}$ the space of $G$-invariant elements in $\wedge^{3} \mathscr{G}$ is one-dimensional.) Thus, if $G$ is one of types $B_{n}(n \geqq 2), C_{n}(n \geqq 3)$ or $D_{n}(n \geqq 4)$, then

$$
\tilde{\eta}_{i k m s}^{j l n p}=D_{m s a}^{n p c}\left(D_{i k c}^{j l a}+D_{k i c}^{l j a}\right)=0 .
$$

Since $\alpha \neq 0$, tensor $\eta_{1234}=0$ does not fulfill Eq. (6.10). This completes the proof of Theorem 3.

Remark. If $G$ is of type $A_{n-1}$, then for the structure tensor $D_{i k m}^{j l n}$ we have

$$
D_{i k m}^{j l n}=\operatorname{tr}\left(f_{i}^{j}\left[f_{k}^{l} f_{m}^{n}\right]\right) .
$$

In this case $D$ is $G$-invariant but it is not an element of $\wedge^{3} \mathscr{G}$. 


\section{Conclusion}

This paper provides a complete classification of graded Poisson Lie structures defined by homogeneous brackets for the case of classical complex Lie groups. Here we indicate the connection of our construction with bicovariant differential calculus on quantum groups.

We consider graded Poisson Lie structures as a useful tool to describe the external algebra formalism on a quantum group. In the non-commutative setting [13] the external algebra $\Omega$ is uniquely defined through the tensor algebra over the bimodule $\Omega^{1}$ of quantum first order differential forms and a natural generalization of the flip automorphism. Note that $\Omega$ is found to be a graded bicovariant algebra.

In our approach we also aimed to construct a graded bicovariant algebra $\Omega$ being the deformation of the external algebra $\Omega_{c l}$ over the cotangent bundle $\Omega_{c l}^{1}$ of a Lie group $G$. We assume that in the semiclassical approximation the product in $\Omega$ is determined by a graded Poisson Lie structure, e.g.

$$
\omega \star v=\omega \wedge v+\hbar\{\omega, v\}+\cdots,
$$

where $\hbar$ is a deformation parameter. Algebra $\Omega$ (with $\star$ product) is uniquely defined by requiring to have a unique basis of ordered monomials. For known examples of graded Poisson Lie structures this construction of $\Omega$ can be carried out completely. For instance, all graded Poisson Lie structures on $S L(N)$ can be quantized in the above sense and the corresponding $\Omega$ is described explicitly in terms of defining relations [8,18]. From this point of view $\Omega$ appears as the quantized algebra.

In Woronowicz's scheme the operator $\mathbf{d}$ of the exterior derivative is introduced by using the extended bimodule method. Bimodule $\Omega^{1}$ with classical dimension $\left(\operatorname{dim} \Omega^{1}=\operatorname{dim} G\right)$ is extended to $\tilde{\Omega}^{1}$ by adding the left- and right-invariant first order form $X$. Geometrically, extensions of the corresponding $\Omega_{c l}^{1}$ are obtained through embeddings: $\Omega_{c l}^{1} \subset \tilde{\Omega}_{c l}^{1}$, where $\tilde{\Omega}_{c l}^{1}$ is the cotangent bundle over a group $\tilde{G} \supset G$. Indeed, bicovariant differential calculi on simple quantum groups $[15,16,25]$ are related with graded brackets on $G L(N)$ that are bicovariant (but non-Poisson) with respect to the action of a simple $G$ [9]. The operator d defined on the extended algebra $\tilde{\Omega} \supset \Omega$ as the graded commutator: $\mathbf{d}=[X, \cdot]$ obeys the Leibnitz rule. The last formula has a direct analogue for the case of graded Poisson Lie structures associated with $G L(N)$. Namely, the classical exterior derivative $\mathbf{d}$ can be expressed as an internal object of a Poisson structure: $\mathbf{d}=\{X, \cdot\}$. Moreover, due to the Jacobi identity d obeys the Leibnitz-like rule with respect the corresponding bracket:

$$
\mathbf{d}\{\phi, \psi\}=\{\mathbf{d} \phi, \psi\}+\{\phi, \mathbf{d} \psi\}, \phi, \psi \in \mathscr{A} .
$$

This implies the general definition of a differential Poisson Lie structure as a graded Poisson Lie structure for which Eq. (7.24) takes place.

Now one can raise a question if graded Poisson Lie structures on simple Lie groups are differential ones. For the case of $S L(N)$, computations [8] reveal the negative answer, which shows the crucial difference between the $S L(N)$ and $G L(N)$ cases [7,22]. Moreover, since the classical d does not admit representation $\mathbf{d}=\{X, \cdot\}$ the corresponding quantized algebras can not be supplied with an operator of exterior derivative obeying the Leibnitz rule [18]. Clearly, this is a price we pay for keeping the classical dimension of $\Omega^{1}$.

The case of other simple groups is more delicate and needs further investigation. By Theorem 3 we have an obstruction to deform the external algebra, which is 
absent in Woronowicz's scheme. This obstruction comes from the Jacobi identity for the bracket of the second order. Evidently, if we do not require this identity, then by virtue of Theorems 2 and 3 the bicovariant bracket exists. We suppose that the Jacobi identity for a bicovariant bracket arises as the semiclassical approximation of the Diamond Condition [28] for corresponding quantized algebras. Note that in Woronowicz's scheme the question about the fulfillment of the Diamond Condition for graded bicovariant algebras on simple quantum groups remains open.

Another problem related to simple groups is to establish the existence of nonhomogeneous Poisson Lie structures. To this end one should classify all $G$-invariant tensors of higher ranks with special symmetry (Theorem 2) and then to check the Jacobi identity.

Note also that results for compact groups do not follow straightforwardly from our scheme and this will be the subject of forthcoming papers.

Acknowledgement. The author is grateful to I. Aref'eva, P. Medvedev, M. Semenov-Tian-Shansky and I. Volovich for useful discussions. This work is supported in part by RFFR under grant N93011-147 and by ISF under grant M1L-000.

\section{References}

1. Faddeev, L.D.: Integrable Models in 1+1 Dimensional Quantum Field Theory. Les Houches Lectures, 1982

2. Drinfel'd, V.G.: Quantum groups. Proc. Int. Congr. Math. Berkley, 1, 798-820 (1986)

3. Semenov-Tian-Shansky, M.A.: Dressing transformations and Poisson Lie group actions. Publ. RIMS 21(6), 1237-1260 (1985)

4. Faddeev, L.D., Reshetikhin, N.Yu., Takhtajan, L.A.: Quantization of Lie groups and Lie algebras. Algebra and Analysis 1, 178-206 (1988) (in Russian)

5. Alekseev, A.Yu., Faddeev, L.D.: Quantum $T^{*} G$ as a toy model for conformal field theory. Commun. Math. Phys. 141, 413 (1991)

6. Kulish, P.P.: Covariant non-commutative differential geometry. Zap. Nauch. Sem. POMI 205, 85-91 (1992) (in Russian)

7. Arutyunov, G.E., Medvedev, P.B.: Quantization of the External Algebra on a Poisson Lie Group. Prep. SMI-11-93 (1993), HEP-TH/9311096

8. Aref'eva, I.Ya., Arutyunov, G.E., Medvedev, P.B.: Bicovariant 3D calculus for $S L_{q}(2)$ from Poisson Lie structures. J. Math. Phys. 35(12), 6658 (1994)

9. Arutyunov, G.E., Medvedev, P.B.: On Poisson Lie structure on the external algebra of classical Lie groups. Prep. SMI-10-94 (1994), HEP-TH/9404068

10. Aref'eva, I.Ya., Arutyunov, G.E., Medvedev, P.B.: Bicovariant differential calculus on quantum groups from Poisson Lie structures. In: Lecture of G.E. Arutyunov at the XXX Winter School of Theoretical Physics in Karpacz, HEP-TH/9406205

11. Brzezinski, T.: Lett. Math. Phys. 27, 287 (1993)

12. Isaev, A.P., Pyatov, P.N.: $G L_{q}(N)$-covariant quantum algebras and covariant differential calculus. Phys. Lett. A179, 81 (1993)

13. Woronowicz, S.L.: Twisted $S U(2)$ group. An example of a non-commutative differential calculus. Publ. RIMS. 23(1), 117-181 (1987)

14. Woronowicz, S.L.: Differential calculus on compact matrix pseudogroups (quantum groups). Commun. Math. Phys. 122, 125-170 (1986)

15. Jurco, B.: Differential calculus on simple quantum groups. Lett. Math. Phys. 22, 177 (1991)

16. Carow-Watamura, U., Schlieker, M.S., Watamura, S., Weich, W.: Bicovariant differential calculus on quantum groups $S U_{q}(N)$ and $S O_{q}(N)$. Commun. Math. Phys. 142, 605 (1991)

17. Isaev, A.P., Pyatov, P.N.: Covariant differential complexes on quantum linear groups. Prep. E2-93-416 JINR, Dubna (1993), HEP-TH/931112 
18. Pyatov, P.N., Faddeev, L.D.: The differential calculus on quantum linear groups. Prep. POMI (1994), HEP-TH/9402070

19. Maltsiniotis, G.: C.R. Acad. Sci. Paris 331, 831 (1990)

20. Manin, Yu.: Notes on quantum groups and quantum de Rahm complexes. Bonn Prep. MPI/91$60(1991)$

21. Sudbery, A.: Phys. Lett. B284, 61 (1991)

22. Tsygan, B.: Selecta Math. Sovietica. 12(1), 75-103 (1993)

23. Zumino, B.: Differential calculus on quantum spaces and quantum groups. Prep. LBL-33249 (1992)

24. Takhtajan, L.A.: Elementary course on quantum groups. PAM\# 120, Univ. of Colorado, 1992

25. Schmüdgen, K., Schüler, A.: Classification of bicovariant differential calculi on quantum groups of type A, B, C and D. Prep. 1-94, Univ. Leipzig (1994)

26. Barut, A.O., Raczka, R.: Theory of group representations and applications. PWN, Warszawa, 1977

27. Weyl, H.: The classical groups, their invariants and representations. IAS Princeton, (1939)

28. Bergman, G.M.: The Diamond lemma for ring theory. Adv. in Math. 29, 178-218 (1978)

Communicated by M. Jimbo 
